\title{
Image in Medical Education: Why, When and How to Use It? A Critical Review of the Literature
}

\author{
Dhouha Bacha ${ }^{1}$, Ghofrane Talbi ${ }^{2}$, Wael Ferjaoui ${ }^{2 *}$, Mejri Atef $^{2}$, Lasaad Gharbi ${ }^{2}$ and \\ Bouraoui Saadia ${ }^{1}$ \\ ${ }^{1}$ Department of Pathology, Mongi Slim University Hospital, Tunisia \\ ${ }^{2}$ Department of General surgery, Mongi Slim University Hospital, Tunisia \\ *Corresponding author: Wael Ferjaoui, Department of General surgery, Mongi Slim University Hospital, Tunisia
}

\begin{tabular}{|c|c|}
\hline ARTICLE INFO & ABSTRACT \\
\hline Received: 幽 February 27, 2020 & Citation: Dhouha B, Ghofrane T, Wael F, Mejri A, Lasaad G. Image in Medical Education: \\
\hline Published: March 06, 2020 & $\begin{array}{l}\text { Why, When and How to Use It? A Critical Review of the Literature. Biomed J Sci \& Tech Res } \\
\text { 26(3)-2020. BJSTR. MS.ID.004343. }\end{array}$ \\
\hline
\end{tabular}

\section{Introduction}

The image is a visual representation of the reality or part of the reality (without being the reality itself) [1]. Since prehistory the image has preceded the text. It is at the origin of the development of all linguistic signs. Nowadays, the image is a polysemic word. Indeed, it may have 2 or 3 dimensions. It can be fixed or animated, colored or not, digital or not, real or virtual. Moreover, the image will make clinical diagnosis without recourse to any doctor, thanks to artificial intelligence [2]. The image is a didactic tool widely (and logically) used in the teaching of medicine. The image in medical pedagogy can be figurative (related to resemblance) or being abstract (related to imagination). We consider an image to be a visual signal that illustrates a concept. It is, therefore, all "nontext". Apart some few narrative reviews reported in the literature, our study seems to be the first (to our knowledge) critical reviews that analyses the contribution of image in medicine. The aim of our work is to carry out a critical review of the literature concerning the interest of the image in the teaching of medicine. Through this review, we try to answer the following questions: Why? How? and when using image as a pedagogic tool in medicine.

\section{Material and Methods}

A comprehensive literature search was done over a period of 10 years from 2000 to 2010. Databases from Pubmed, Google Scholar, The National Library of Medicine (MEDLINE) and the Cochrane Library were searche using the following keywords: image, medical education, multimedia instruction, teaching, learning, pedagogy and the following boolean operators: OR, AND. Articles related to medical education, pharmaceutical education, nursing, dentistry and psychopedagogy were included in our search. The selected articles must answer at least one of the 3 previous questions. They may be published in English or French language. Editorials are excluded. All articles are read and discussed by all authors of this article. Our study was performed according to the Preferred Reporting Items for Systematic Reviews and Meta-Analyses (PRISMA). References are organized using the ZOTERO software.

\section{Results}

30 articles are selected. This is a quantitative study in 20 cases and a qualitative study in 10 cases. 15 articles answer one of the previous questions, 10 answer two questions and only 5 articles answer all three questions. The disciplines concerned are medicine, pharmacy, nursing, dentistry and pedagogy. The impact factor of the journals publishing the selected papers varies from 0.3 to 1.5 (SCIMAGO).

\section{Image in Medical Education: Why is this?}

Image enhances all domains of learning which is divided into 3 categories: cognitive domain (knowledge), affective domain (attitudes) and psychomotor domain (skills) [3]. It improves the 3 stages in the memory process: encoding, storage and retrieval. These 3 stages are intricate. Indeed, the brain must encode information 
into a storable form so that it can be recalled later [4]. Furthermore, encoding via an image is better and faster than encoding via a word or text. That is why the displaying of auscultator data (visual coding) in cardiology, for example, is better in diagnostic than isolated auscultation (acoustic coding). The image makes, in this example, encoding through 2 ways (visual and acoustic). Indeed, interpreting an image aloud allows the brain to process information using visual and acoustic coding. Similarly, drawing an image while touching it (a bone in anatomy education, for example) is another example of double coding through visual and haptic paths.

On the other hand, long-term memory encodes new fact and information semantically for storage as demonstrated by Baddeley [5]. An image needs, therefore, to enter into visual working memory before being stored onto long-term memory. Moreover, pictures and images are more likely to be remembered than words and texts. That's what we call "picture superiority effect" which is demonstrated in various studies [6]. Thus, an image transmits a maximum of educational messages in a minimum of time and space. The use of color-coding increases memory efficiency. Memory enhancement is achieved through repetition and active storage

However, the superiority of a video compared to a still image is enhancing memory isn't yet demonstrated. Beyond memorization, the image is a useful tool to help both sequential learners who need to learn in steps (via sequential images) and global learners [7].

In addition to memorization, the image improves reasoning. It is a process which inputs are specific information and outputs are consequent decision. When the input is an image, reasoning is called visual reasoning. This visual reasoning reinforces critical vision and meta-cognition through two strategies: Visual Thinking Strategies (VTS) and Art Thinking (AT) [8]. In VTS, the learner is asked to answer the following 3 questions:

a) What's going on in this picture?

b) What makes you say that?

c) What else can you find?

AT is a method similar to VTS but is aimed at artworks, plays or films. The usefulness of AT in learning (affective domain) is demonstrated. Otherwise, The Eye Tracking method evaluates this visual reasoning. It is a quantitative process measuring either the point of gaze where one is looking or the motion of an eye relative to head [9]. Finally, the image is a tool appreciated by learners, especially if it is used through social networks.

\section{Image in Medical Education: How Can it be Used?}

The use of images is mandatory in several methods because it is the foundation on which they are based (CIAP (Clarification, Illustration, Application, Participation)-Mini Talk Chalk) [10]. The other methods use it widely (60\% in reading) despite the optional character of this choice. Short term methods (Micro-
Mini teaching), for example, use the image because of its PSE and because It fits perfectly in playful teaching methods (via cartoon images for example). Several disciplines are perfectly suited to teaching through images. These are mainly surgery, radiology, dermatology, anatomopathology and anatomy. Indeed, the image is the primary tool in teaching the anatomy of the "living human" via: imagery (Computed tomography scan and/or Magnetic Resonance Imaging), body painting and video capsules of endoscopic surgery (Figure 1). Whatever the method (and the discipline), the choice of a given image depends on the pre-established educational goals in a perfect alignment between objectives, methods and evaluation. It can be a still, sequential or animated image. It can be real, virtual or both (in augmented reality).
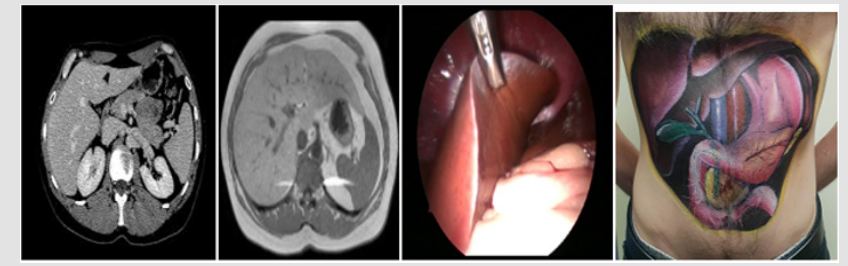

Figure 1: Image used in teaching anatomy (example here of the liver) may be radiological (A, B), laparoscopic (C), body painting (D).

Faced with a still image, the learner can be brought to:

a. Identify (part or the entire image).

b. Interpret (in a clinical or ethical context).

c. Draw (or color) part or the entire image. Indeed, Drawing allows the mental image of the learner to be recovered.

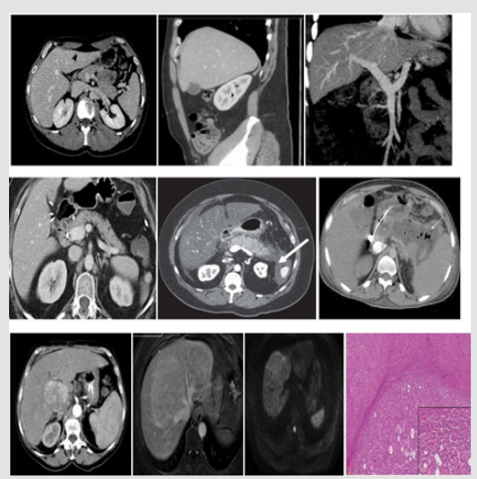

Figure 2: 3 examples to illustrating the concept of sequential image.

Faced with a sequential image (Figure 2) the learner can be led to:

I. Iinterpret a sequence.

II. Arrange a sequence in disorder (in time or space).

III. Draw a missing image in a sequence (or lengthen a sequence). 
Indeed, the sequential image allows teaching by analogy or concordance and promotes mental rotation.

In front of a video the learner can be led to:

i. Interpret.

ii. Produce a video of a clinical case.

iii. Produce a video selfie, mirror effect.

Elsewhere, the image may be a symbol or a graphic (Figure 3). The image is, thus, a mediator of action, co-action and interaction. Action-coaction-interaction, indeed, improves learners' motivation and enables them to detect their errors in order to remedy them through feedback action. This trilogy (action-coaction-interaction) enables the image to meet the requirements of the different teaching methods (active pedagogy, participatory pedagogy, collaborative pedagogy).

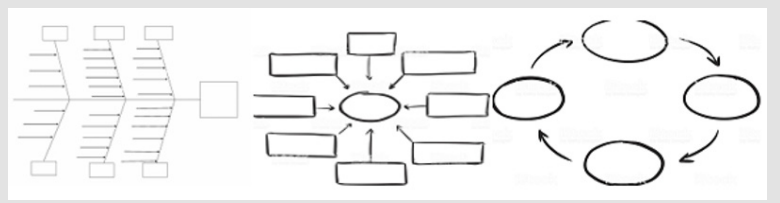

Figure 3: Image in medicine may be also symbol pr graphic or diagram.

\section{The Image in the Teaching of Medicine. When is it used?}

An image can be used before the course (video capsule as part of a reverse pedagogy or video tutorial).

During the course, the image achieves different objectives depending on the teacher's scenario, as follows:

a) At the beginning of the course a shock image attracts the attention of the learners by creating an emotionally strong moment (or light bulb moment).

b) In the middle of the course an image can signal a change of direction in the presentation (or signposts).

c) At the end of the course an image can be used to reframe the concept(s) taught (thanks to big picture).

\section{Discussion}

Like any teaching tool, the image has the above-mentioned advantages, but it also has its pitfalls. Indeed, the image can lead to errors of perception (gestalt). Moreover, it can be restrictive of the concept which is meant to illustrate. Finally, it only affects visual learners in the VARK model (Visual, Auditory, Read/Write, and Kinesthetic) [11].

In order to avoid these pitfalls, we recommend three modes of round trip:

a) Within the image, round trip from the concrete to the abstract (or from the fixed to the animated).

b) Within the tool, round trip from the image (non-text) to the text.

c) Within the method, round trip from the pictorial methods to those that are not pictorial (bedside teaching).

\section{Conclusion}

In today's highly image-based world, the use of images in medical education is essential. Indeed, the image is a pedagogic tool highly recommended. However, the advantages and disadvantages of such a tool must be known by all teachers.

\section{References}

1. (2020) IMAGE | meaning in the Cambridge English Dictionary. Disponible sur.

2. Khodaskar A, Ladhake S (2015) Semantic Image Analysis for Intelligent Image Retrieval. Procedia Computer Science 48: 192-197.

3. (2020) Three Domains of Learning-Cognitive, Affective, Psychomotor. The Second Principle. Disponible sur.

4. Stangor C, Walinga J (2014) 9.1 Memories as Types and Stages. In: Introduction to Psychology-1 ${ }^{\text {st }}$ Canadian Edition. BCcampus. Disponible sur.

5. Baddeley AD (1966) The Influence of Acoustic and Semantic Similarity on Long-term Memory for Word Sequences. Quarterly J Experimental Psychology 18(4): 302-309.

6. Defeyter MA, Russo R, McPartlin PL (2009) The picture superiority effect in recognition memory: A developmental study using the response signal procedure. Cognitive Development 24(3): 265-273.

7. Brem AK, Ran K, Pascual Leone A (2013) Learning and memory. Handb Clin Neurol 116: 693-737.

8. Hailey D, Miller A, Yenawine P (2015) Understanding Visual Literacy: The Visual Thinking Strategies Approach. Essentials of Teaching and Integrating Visual and Media Literacy pp. 49-73.

9. Lupu R, Ungureanu F (2013) A survey of eye tracking methods and applications. Bulletin of the Polytechnic Institute of Iaşi Automatic and Computer Section P. 3.

10. Kumar N (2018) Importance of Talk and Chalk Method of Teaching: Dental Students' view in Preclinical Years. International Journal of Healthcare Education \& Medical Informatics 5: 11-5.

11. Hussain I (2017) Pedagogical Implications of VARK Model of Learning. 


\section{ISSN: 2574-1241}

DOI: $10.26717 /$ BJSTR.2020.26.004343

Wael Ferjaoui. Biomed J Sci \& Tech Res

\section{(C) (P) This work is licensed under Creative}

Submission Link: https://biomedres.us/submit-manuscript.php

\begin{tabular}{ll} 
BIOMEDICAL & Assets of Publishing with us \\
RESEARCHES & - Global archiving of articles \\
& - Immediate, unrestricted online access \\
& - Rigorous Peer Review Process \\
\hline
\end{tabular}

